\title{
Philosophiques
}

\section{Ex ante ou ex post : comment l'éducation et la formation professionnelle modifient-elles la juste part ?}

\section{Peter Dietsch}

Volume 41, numéro 1, printemps 2014

URI : https://id.erudit.org/iderudit/1025728ar

DOI : https://doi.org/10.7202/1025728ar

Aller au sommaire du numéro

Éditeur(s)

Société de philosophie du Québec

ISSN

0316-2923 (imprimé)

1492-1391 (numérique)

Découvrir la revue

Citer ce document

Dietsch, P. (2014). Ex ante ou ex post : comment l'éducation et la formation professionnelle modifient-elles la juste part ? Philosophiques, 41(1), 161-164. https://doi.org/10.7202/1025728ar d'utilisation que vous pouvez consulter en ligne. 


\title{
Ex ante ou ex post: comment l'éducation et la formation professionnelle modifient-elles la juste part?
}

\author{
PETER DIETSCH \\ Université de Montréal
}

Des PDG qui rapportent des millions de dollars; des vedettes de sport ou de musique dont les contrats continuent de battre tous les records; et même le "simple» banquier ou avocat qui peut espérer obtenir un bonus dans les sept chiffres en plus de son salaire de base. Est-ce que ces rémunérations sont justes? Y a-t-il une justification morale ou économique pour ces salaires astronomiques, et notamment pour l'écart entre ceux-ci et le salaire d'un «simple» travailleur?

La juste part de David Robichaud et Patrick Turmel illumine un aspect central du débat sur la justice salariale - un aspect qui, de manière surprenante, échappe à la plupart des contributions abordant la question sous un angle économique. Celles-ci supposent que le salaire d'un travailleur reflète sa productivité marginale, c'est-à-dire l'ajout à la production que son emploi génère. Dans cette logique, c'est la contribution importante de certains individus qui justifie leurs salaires extraordinaires. Pour citer un exemple classique, le succès de General Electric dans les années I 980 à 2000 était attribué aux compétences de gestion de son PDG Jack Welch. Visiblement, une partie importante de la valeur créé par l'entreprise s'expliquait par l'influence de celui-ci, ce qui justifiait le salaire élevé qu'on lui payait.

Robichaud et Turmel font valoir que cette manière de présenter les choses néglige les effets de réseaux dans la production économique. Bien que les compétences d'un Jack Welch jouent certainement un rôle, le facteur décisif dans la création de valeur est la coopération entre une collectivité de travailleurs spécialisés. La hausse de productivité générée par la division des tâches est un thème qui a une longue histoire dans la pensée économique. La manufacture d'épingles d'Adam Smith fournit l'illustration classique des gains de la division du travail. La question que Smith ne développe pas encore est celle des conséquences distributives de la division des tâches.

Comme Robichaud et Turmel, j'estime que la prise en considération des effets de réseaux a des implications importantes pour notre théorie de la justice économique. Dans un autre texte, je défends en détail l'idée que le surplus coopératif généré par la division des tâches devrait être distribué de façon égalitaire ${ }^{1}$. Robichaud et Turmel utilisent la production d'un grille-

1. Voir Peter Dietsch, "Distributive Lessons from the Division of Labour", Journal of Moral Philosophy 5/I, 2008, 96-I I7.

PHILOSOPHIQUES 41/1 — Printemps 2014, p. 161-164 
pain pour traiter la même intuition. Le fait que personne ne serait capable de fabriquer à lui seul un produit relativement simple comme un grille-pain met en évidence la taille énorme du surplus coopératif. Ce n'est pas seulement la coopération à l'intérieur de la firme qui produit les grille-pains, mais la coopération plus large dans la société, et même à travers le monde, qui contribue à ce surplus. Comme Robichaud et Turmel le soulignent, attribuer la valeur créée à une seule personne, comme le PDG, ou à un petit groupe de personnes, comme l'équipe de gestion, construit des mythes de «crusoéconomique " et de production autarcique qui ne correspondent pas à la réalité. Comme Adam Smith le percevait déjà à son époque, «dans la réalité, la différence de talents entre les individus est bien moindre que nous le croyons, et les aptitudes si différentes qui semblent distinguer les hommes de diverses professions quand ils sont parvenus à la maturité d'âge, n'est pas tant la cause que l'effet de la division du travail, en beaucoup de circonstances ${ }^{2}{ }^{»}$.

\section{L'objection du caractère de la remplaçabilité différentielle}

Ayant dessiné les contours de l'argument central de Robichaud et Turmel, je consacrerai la plus grande partie de ce texte à la formulation de l'objection que je considère la plus difficile à écarter pour quelqu'un qui, comme moi, est très sympathique à la position défendue dans La juste part.

L'objection en question repose sur la distinction entre les talents naturels des individus au regard de leurs compétences acquises par l'éducation et la formation professionnelle: il est vrai que les talents naturels ne varient pas tant entre individus. Mis dans une situation d'autarcie, Jack Welch ne serait pas beaucoup plus (ou moins) productif que d'autres individus ${ }^{3}$. Par contre, l'éducation, la formation et l'expérience professionnelle font en sorte que la qualité des compétences acquises par les individus varie énormément. La connaissance approfondie que Jack Welch a de General Electric comme entreprise ainsi que son expérience de gestion le rendent très difficile à remplacer. Pour prendre un exemple à l'échelle sociale et non à l'intérieur d'une entreprise, une chirurgienne cardiaque est beaucoup plus difficile à remplacer qu'un balayeur de rue. Bien que la chirurgienne ait fort probablement plus de talent que le balayeur, la différence entre leur talent est moindre que la différence entre leurs compétences acquises respectives.

La rémunération sur le marché du travail suit les compétences acquises et non les talents naturels. Donc, même si l'on admet que le surplus coopératif est important et dépasse largement ce que les individus pourraient produire en autarcie, on peut défendre des inégalités salariales importantes en faisant appel à la remplaçabilité différentielle des individus. L'argument de

2. Adam Smith, Recherches sur la nature et les causes de la richesse des nations, I776, livre I, chapitre II.

3. Évidemment, cet énoncé ne tient pas pour des individus gravement handicapés. Pour l'argument qui nous concerne ici, nous pouvons mettre ces cas de côté. 
Robichaud et Turmel néglige cet aspect. Si l'objection est bien fondée et si la remplaçabilité est un déterminant légitime du salaire d'un individu, le surplus coopératif devra être distribué d'une façon inversement proportionnelle à la remplaçabilité de l'individu en question. Autrement dit, reconnaître l'importance des effets de réseaux dans la production économique n'implique pas une position égalitariste pour ce qui est de la distribution du surplus coopératif.

Que pourraient répondre Robichaud et Turmel à cette objection? La première stratégie de réponse est de demander combien de personnes sont à ce point difficiles à remplacer qu'une prime de salaire importante soit justifiée. Comparons une chirurgienne cardiaque et un PDG. Dans le cas de la chirurgienne, il est vrai que la formation d'un nouveau médecin prend beaucoup de temps et de ressources. C'est la spécificité du capital humain qu'elle a accumulé qui fait qu'elle est difficile à remplacer. À première vue, on pourrait croire que la situation du PDG est similaire. Par contre, on observe aujourd'hui que le marché du travail pour les PDG est assez large. Des gestionnaires occupent successivement des postes dans différentes entreprises, passant même d'un domaine à l'autre, ce qui suggère que leur capital humain n'est pas aussi spécifique que celui de la chirurgienne cardiaque. Dans ce cas, si la remplaçabilité est le déterminant principal du salaire, celui de la chirurgienne devrait être plus élevé que celui du PDG, ce qui, de manière générale, n'est pas le cas aujourd'hui. Par conséquent, faute d'une autre justification pour cette inégalité entre les deux professions, l'argument de Robichaud et Turmel pourrait encore s'appliquer, du moins en partie.

Notons par contre que cette première stratégie de réponse fait une concession importante à l'objection de la remplaçabilité différentielle. Elle admet que la remplaçabilité est un déterminant légitime du salaire. La deuxième stratégie de réponse consiste à nier cela. La question de la justice salariale, pourraient faire valoir Robichaud et Turmel, n'est pas une question de négociation de laquelle celui qui a des compétences rares peut extraire un salaire plus élevé que les autres. Un tel énoncé semble en contradiction directe avec toute explication du salaire en sciences économiques. Mais justement, pourraient continuer Robichaud et Turmel, ces explications négligent les effets de réseaux discutés plus haut. Si la personne en question n'a pu acquérir les compétences indiquées plus haut que grâce à la coopération des autres, alors ses droits aux fruits de leur rareté seront limités. Encore une fois, la distinction entre talents naturels et compétences acquises devient pertinente à ce stade-ci. S'il s'agissait de talents naturels, on devrait accepter les prémisses de l'égalitarisme libéral moderne - notamment l'idée que toute inégalité non méritée doit être compensée — pour défendre la position que l'individu en question n'a pas droit aux fruits de son travail. Mais comme il s'agit de compétences acquises, nous ne sommes pas contraints d'endosser l'égalitarisme libéral pour dire que ni le PDG ni la chirurgienne cardiaque n'ont un droit illimité aux fruits de «leur» travail. Ce droit serait 
limité, parce qu'une fois que l'on tient compte des effets réseaux dans la production, ce ne sont pas entièrement les fruits de leur travail, mais les fruits de la coopération sociale.

La question fondamentale qui sépare donc Robichaud et Turmel des partisans de l'objection de la remplaçabilité différentielle est la suivante: jusqu'à quel point la remplaçabilité ou non d'un individu est-elle un facteur qui relève de l'individu lui-même? N'est-elle pas plutôt un facteur qui relève du rôle que cet individu joue dans la coopération sociale? Si le rôle en question en est un que l'on peut seulement remplir après avoir acquis un capital humain très spécifique, le fait que l'individu qui remplit ce rôle est difficile à remplacer relève - du moins en partie - du rôle, et non de l'individu. La partie du surplus coopératif en question reviendrait donc à la collectivité, et non à l'individu.

Dans cet article bien succinct, il nous sera impossible de trancher cette question fondamentale. Je conclurai en soulignant encore une fois l'importance du débat que Robichaud et Turmel ont suscité avec leur livre. La juste part n'apporte pas seulement une contribution à la discussion académique, mais le livre possède également la rare vertu de vulgariser ces questions de justice distributive pour un public plus large. 